\title{
Design and Implementation of Bandwidth Monitoring, Line Aggregation of VoIP
}

\author{
Tun Tun Oo, Aaron Don M. Africa \\ Department of Electronics and Communications Engineering \\ De La Salle University, Manila \\ 2401 Taft Ave., Malate, Manila 1004, \\ Philippines, tuntunoo@dlsu.edu.ph, aaron.africa@dlsu.edu.ph
}

\begin{abstract}
Nowadays, there are many ways to get the Internet connection such as ADSL, Mobile, Wifi, WiMax, Fttx. Furthermore, the Internet connection can get anywhere and any times. In fact, the PBX systems would be low interested because the PBX (Private Branch Exchange) is used circuit switching system and is also used to expend the phone bills [1]. The VoIP system uses the packet switching which it can use everywhere in the place of getting an Internet connection. Moreover, the Phone bill costs are lowest and flexible to use in the new era. In this paper, the VoIP technology is used bandwidth control and line aggregation to get the network speed in the network infrastructure. Moreover, the traffic and bandwidth of each port and whole systems can monitor and manage. In this paper, the OSPF, Ether channel and bandwidth monitoring system are used in real time simulations. All of systems are used the Cisco devices, Linux and windows OS and line aggregations. Line aggregation means line compositions. One switch port can use $100 \mathrm{Mbps} / 1 \mathrm{Gbps}$. On the other hand, if the port is 1Gbps, the four ports are used that it may be $4 \mathrm{Gbps}$. Moreover, not only line combination, but also link redundancy. In this paper, the Gns3 simulation is mainly emulated in the virtual environment with VMware. GNS3 (Graphical Network simulator) allows simulation of complex network structure and the outside world. It can interoperate and communication together with VMWare workstation, Virtual Machine and Esxi to imitate various OS in a virtual community such as Linux, Windows OS and Cisco IOS. GNS3takes this a step further by providing a graphical environment.
\end{abstract}

Key words : ADSL; OSPF; PSTN; PBX; VOIP, WiMax, GNS3, ESXi

\section{INTRODUCTION}

The network infrastructure of the PSTN is selected the outside or locally by the exchange. Exchange of PSTN uses circuit switching. The exchange will select an outside (trunk) or local call until they spanned all over the world coverage via copper cables, microwaves, optical fiber cables and satellite communication. PSTN network is a very old network and cause the many errors [2]. Copper cable of PSTN network has limitation and many of cable is used for each user. So, the connected many copper cable networks are not looking good for the landscape beauty of country or cities.

Cisco devices are used all over the world that they are led in the backbone network and delivering a seamless integration of technology innovation, hardware platform support, and business critical services. Cisco operates their own operating system that it is called IOS (Internet Operating System). It is a complex operating system optimized for Internetworking that it is separate from the hardware. The user runs the man-machine interface to perform function setting on the network device. The functions provided are as follows: the function, preference setting of the network device and the connection port, and the data transmission and security managements setting between the running network protocol and the network function device.

This paper has three network layer models such Core, Distribution and Access layer for large business into a two layer network model (Core/distribution and access layer) for small business. Indeed, the core switches will distribute via the voice gateways and configured to automatically route calls from a voice gateway to other systems. The technology of VOIP (Voice Over Internet Protocol) has changed the our life's work and collaborate. Furthermore, a redundant design is examined specifically for the large business enterprise network for increased reliability and technological evolution. This paper has also implemented the EtherChannel technology. EtherChannel is a ports and link aggregation technologies, advanced by Cisco that it is supplied fault-tolerant highest data speed links between Switches, Routers, and Servers. EtheChannel technology allows multiple physical Ethernet links to join into one logical channel.

\section{LITERATURE REVIEW}

The previous telephone network system has been taken over from by soft switch or digital switching like VoIP services because of Internet progressing. The customer should have the knowledge of networking and telephone system. VoIP lab intends for adjustable configuration to grant the implantation for many applications. [1] The advantage of Cisco devices of a VoIP lab qualifies the student's knowledge to obtain a reliable system. The more performance network is provided Open Shortest Path First and Multi-Protocol Label Switching 
(MPLS) to obtain Quality of Service and security of the application of VoIP system. There are three OSPF areas that they are one backbone and two standard areas. In this system, the proxy server is located to operate proxy as I-CSCF, S-CSCF, P-CSCF. [2] Moreover, the performance of VoIP is considered bandwidth, packet end to end delay, jitter and packet deliver. Traffic Engineering should be considered the Qos, Speed of VoIP, MPLS and redundancy. [3] Cisco routing and Asterisk base implementation of Public Branch Exchanged (PBX).[4] The future work will routing information protocol such as OSPF, RIP, EIGRP, VPN tunnels, IP-Phone and Multiple network MPLS. [5] Soft-switch or VoIP communication system has simplified, openness and flexibility that it is also provided the devices on the market such as Cisco IP phone, another IP Phone, media gateway to reduce the cost of the system greatly. [7] The transmission and receiving technology of voice and data via the Internet have been operated to assess the performance of VoIP between different networks. Simulation modeling and lab experiment are considered very important to use the difference or multipoint network.[8] The VoIP network obtains the services a router that combined the network cloud services, by the internet to the network. When the secure network with VoIP, the firewall system is used that it is connected to the server. In fact, the network will increase speed, throughput, data transfer, delay, packet transfer.

When the author expands VoIP calls into the WAN (Wide Area Network) and MAN (Metropolitan Area Network) space, the author should also regard the difference between VoIP calls that come from other Cisco Unified CME nodes. The authors WAN (Wide Area Network) and MAN (Metropolitan Area Network) network versus VoIP calls that are from VoIP Public Switched Telephone Network (PSTN) gateways or even from other independent external/wholesale VoIP carrier networks. This Cisco 8211CME (Call Manger Express and Cisco 3825 ISR (Integrated Service Router) router can link separate VoIP networks together and participate into the VoIP network using IP (Internet Protocol) to IP (Internet Protocol) gateways.[9] This arrangement may be desirable if the VoIP network wants to communicate international and long-distance phone service directly from a carrier-class VoIP service provider and have this linked at the VoIP level to private business or enterprise VoIP network.

\section{THEORETICAL CONSIDERATION}

In designing of VoIP, the main requirement is to have a system which call the IP-phone from every phone via the network structure. These systems mainly need for more bandwidth and efficient connectivity. This is also needed to know cable management system and IP addressing problem in the VoIP system. And then also know how to go the packet, and frame structure between router and switches. This paper is significance for data redundancy and line aggregation (increase bandwidth) of VOIP (Voice Over Internet Protocol). The data redundancy is also called HSRP (Host Standby Routing Protocols). HSRP is the standard of Cisco of supporting the path of high data speed network availability. This is supported first hop redundancy for Internet Protocol (IP) host. IEEE 802
LAN is configured with a default gateway IP address. IP traffic without relying on the availability of any single router is routed by HSRP. It enables a set of routers to operate with the appearance of a single virtual router or the default gateway to the nodes via a Local Area Network (LAN). If HSRP is configured on a segment of the network, it will support physical address (MAC) and logical Address IP that it is distributed among a group of networks or routers. Physical Address and logical address of a virtual router is allowed two more HSRP configuration routers to use MAC address. The virtual routers are configured to provide round robin or alternate to each other, such as active and standby. One of the routers is choice to be the active router and the other router is standby router because one router of the group is failed that while another router is suddenly operated within $2 \mathrm{Sec}$. This paper has discovered the problems that they are VOIP IP phone number assign problems, OSPF (Open Shortest Path Fast) routing Problem, Spanning Tree Protocol problem and Voice called connection problems via different networks. The OSPF network diagram is shown in figure 1.

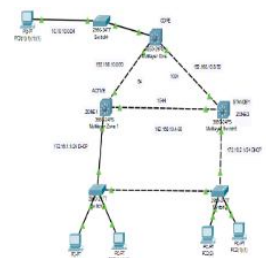

Figure 1: The OSPF Network Diagram with bandwidth

The purpose of this paper is to solve the impact of Voice Over Internet Protocol (VOIP). And then, the background knowledge on VOIP service will be provided. This problem has important things that they will be matched mac-address and ephone number assign.

The goal of this thesis is to create within VoIP systems and Cisco 2811 CME or Cisco 3825 ISR router is the network infrastructures a new, efficient model for providing reliable VoIP with scalability, security and QoS considerations. To accomplish the goal, it is necessary to: Firstly Problem, STP is conflicted between switch upon the network infrastructure in the network switch or bridge.[10] The requirement of the Spanning Tree Protocol (STP) proceeds because switches in local area networks (LANs) are often interconnected using redundant links to improve resilience should one connection fail. However, this connection configuration creates switching loop resulting in broadcast radiation and forwarding information base instability. [11] If redundant links are used to connect switches, then switching loops need to be avoided. The second problem is routing and switching that it is decided the data to warranty reach the real destination address. Third Problem, this problem has important things that they will be matched mac-address and Ephone number assign. Fourth Problem is Voice Called Connection Problems via Difference Networks and line aggregation and data redundancy.

Fast EtherChannel grants multiple physical fast Ethernet links to associate into one logical link channel. In the channel, the traffic load sharing is permitted the redundancy that one or more links in the channel breakdown. Interconnect LAN, 
routers, servers and workstation can use fast Etherchannel via UTP or fiber cable. The concept of load balancing and redundancy use the Etherchannel technique. There are three protocols in Etherchannel. They are PAgP (Cisco propriety), LACP (IEEE standard 802.3d) and Load balancing. The mainly important issue are following to use Etherchannel:

1. All ports are same duplex

2. All ports are same speed

3. All ports are same native VLAN

4. All ports are same access port or trunk port

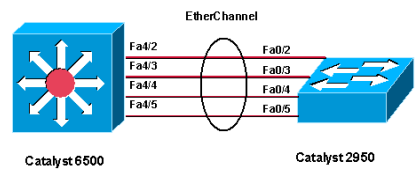

Figure 2: Etherchannel on Cisco Switches

Table 1: Recommendation Protocol Mode in LACP

\begin{tabular}{|c|c|c|c|c|}
\hline & On & Active & Passive & Off \\
\hline On & Yes & No & No & No \\
\hline Active & No & Yes & Yes & No \\
\hline Passive & No & Yes & No & No \\
\hline Off & NO & No & No & NO \\
\hline
\end{tabular}

The difference network phone call system is carefully controlled to each difference network infrastructure because this issue is very important to call VoIP voice via different networks. The solution of the VoIP voice calls via difference network command.

Router(config)\#dial-peer voice 9100 voip

Router(config-dial-peer)\#session target ipv4:172.16.1.3

Router(config-dial-peer)\#destination-pattern 91..

\section{DESIGN CONSIDERATION}

Router have two ports that it is LAN port and the WAN port. LAN port is used for Router, Local Area connection that it is also called internal connection. WAN port is connected to the outside world that it is an Internet connection or other difference network. In this router, the sub interface LAN function is used the sub VLAN, Truck and DHCP function. DHCP means that Dynamic Host Configuration Protocol that it uses auto IP assigned to the switch and IP Phone. And then it is also configured to match IP address and MAC address that it is also called physical address and logical address.

Figure 3 shows the flow of the implementation of a CME Router network of the difference VoIP system techniques used for the whole VoIP system. In this network model system, three c7200 router for OSPF routing, Four L3 switch for HSRP and two main telephone network group. Three group number systems are used in this paper such as $71 .$. , $91 \ldots$ and $09666 \ldots .$. In $91 \ldots$ network, the voice router (CME) is used to call the phone call and L3 switch is used for VLAN 10 and 20 (Ip 192.168.10.0/24 and 192.168.20.0/24). In addition, the ether channel and spanning tree mode are used to improve bandwidth and redundancy. The system of 09666 phone network system is used same way as $91 \ldots$ network. Even though, the different VLAN is used such as Vlan 30 and Vlan40 is used.

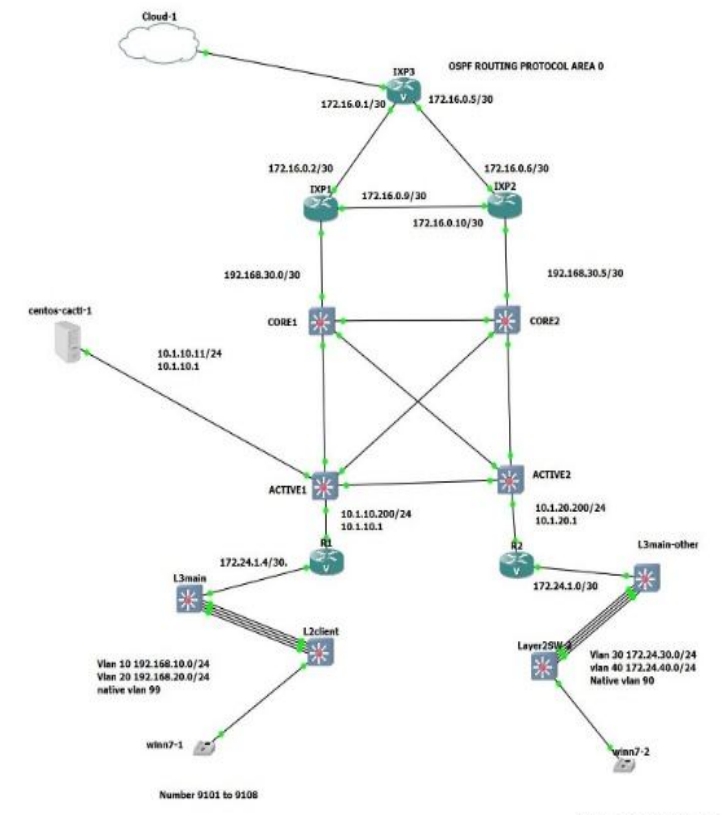

Figure 3: The network infrastructure of VoIP in GNS3

The Cisco ISR routers are used for call manager express of VoIP to communicate voice and data packet. It is advanced to secure the Voice, Data, video services. Cisco ISR also supported the network agility, intelligence and performance and embedded the security processing, high memory capacity and high density interface. It can accept the telephony services. However, the IP-phone Mac address and IP address are matched. This problem is the challenge of this system. The Cisco can also separate sub interface LAN with one LAN Cable. However, the author has not used this method because the telephone service and sub interface LAN may complex and delay the systems. So that, the authors design the telephony service commands. Another device is used two L3 Switch that it is Cisco C3560 -24 catalyst switch. Cisco C3560 24 Catalyst Switch has 24 ports and can separate VLAN (Virtual LAN) or sub LAN because the L3 switch operate not only switch options but also router options. L3 Switch router options are better than router option because the latency of 13 switch is faster than the latency of routers. Its' service includes IEEE 802.3af and PoE (Power on Ethernet). The deployment of Cisco L3 switch is used the new feature such as wireless access, video surveillance measuring and IP telephony at the enterprise network. Moreover, the L3 base IP base software that it can configure the IP assigned and DHCP (Dynamic Host Configuration Protocols) services. In fact, VLAN 10, 20 and 30 services are interoperated in Cisco 3560 Catalyst Switch. Cisco WS C2960 24 port catalyst switch is used in the infrastructure. It is intended for the VLAN trunk, access option and ether-channel or Line aggregation. Cisco C2960 Switch is L2 switch. So that, it could not operate router functions like IP assigned and DHCP function. The other is used Multilab software and Cisco IP Communicator. Multilab software and Cisco IP communicator installed in the Window OS such as 
Window 7 and Window 10 system. The multilab software operates multi IP telephone system and multi assign mac-address of the IP phone. In fact, the various IP phone can communicate as same operating system. The author is a specific call system for difference network via HSRP and OSPF network infrastructure. And then, It provides the multiple calls per directory number (DN) and hold, transfer and call conference option. The future design of VoIP will support camera or video option. So that, the next generation of IP phone can communication the video call system to each other. In this design, the GNS3 simulator is used commonly that it can operate outside world and VM simulator. But, It has many requirements to use such as CPU, RAM and Hard disc capacity. Another more, monitoring system is used in this system like Cacti monitoring system to get the traffic of each interface.

\section{METHODOLOGY}

Two L3 Switches, two L2 Switches and six VoIP IP-phone. Cisco router C3825 ISR is used in this system due to VoIP services because other routers (not ISR or CME) do not support the Voice services. Cisco devices are used in this infrastructure because they are secure and high performance of the network backbone system. All of Cisco devices are operated Cisco IOS software that they lead network software, delivering integration of technologies innovation for business and enterprise network infrastructure. The SOHO network to core network system is leveraged the Cisco IOS widely. The CLI (Command Line Interface) differs from other network operation systems.

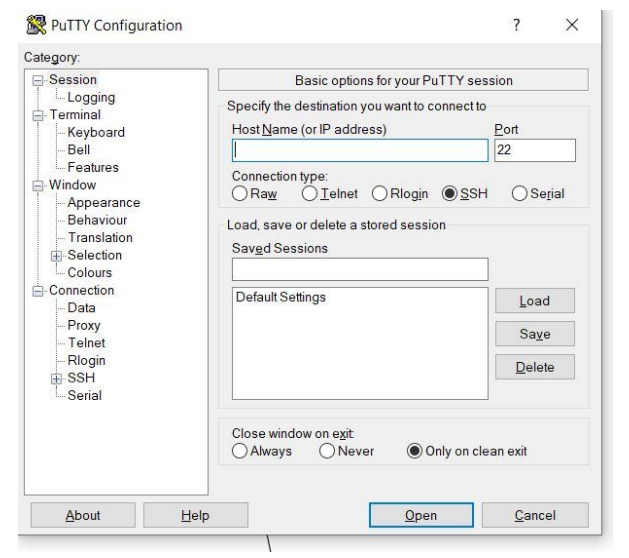

Figure 4: Putty Cisco Configuration Software

The Putty Cisco configuration software as shown in Figure 4. If the author wants to configure the Cisco devices, the Putty software should use. PuTTy is a compatible terminal for Windows OS that it is the most popular SSH client at the open source program. It can support SSH (Secure Socket Shell), telnet and supports authentications too. Its' terminal is same GUI or CLI of Cisco internal IOS system. The Putty software open and connect with computer and Cisco routers and switch via USB (Universal Serial Bus). And then, the $\mathrm{SSH}$ radio box is selected and the port 22 automatics appear in the second box because the SSH port service is used Port 22. When the first time connect to the Cisco routers or switches, it will prompt the user and password authentication to accept SSH server key. Telnet is also operated like SSH. However, Telnet Port is 23. Bandwidth and Traffic Monitoring system is used that it is a Cacti monitoring system. It is a free (open-source), graphic tool and web-base monitoring designed application that it is data logging tool RRDtool. Cacti displays and predetermine the time interval and graph the resulting data that it is speed, the CPU condition of network devices, bandwidth utilization, jitter. Simple Network Management Protocol (SNMP) is used for network traffic monitoring by polling the Cisco routers or Cisco switches. The open source OS that they are REDHAT, CentOS, Fedora Linux can install the Cacti. However, APache (Web Service), MySQL (Database server), PHP (script module for graph), SNMP and RRD tool (bandwidth, speed and CPU)[12].

The data are analyzed once all the data have been gathered and the algorithms used have been successfully applied under the correct experiment set-up. After the said phase, the data retrieved during the experimentation will be compared to Data or packet going to each equipment. Other important information such as IP phone call to each other, packet distribution system to each phone number group and data flow will be gathered. For this, the experimental parameters such as phone calling system and line redundancy facility, data communication, DNS server and system reliability will be tested. The condition of the speed and bandwidth are used in the Cacti Monitoring system. The traffic and bandwidth analyzed to use the Cacti monitoring system. The test result manages and monitoring call traffic bandwidth, switch to switch ether-channel line condition monthly. In this paper, the Gns3 simulation is mainly emulated in the virtual environment with VMware. GNS3 (Graphical Network simulator) allows simulation of complex network structure and the outside world. It can operate and communication together with VMWare workstation, Virtual Machine and Esxi to imitate various OS in a virtual community such as Linux, Windows OS and Cisco IOS. GNS3takes this a step further by providing a graphical environment

\section{RESULTS AND DISCUSSION}

The VoIP phone call in same network is shown in figure 5. The system of network infrastructure is misled by various problem to communicate with each other in GNS3. The interoperate of GNS3 and VMware with cacti and window OS is very difficult to handle to each other. However, the Cisco router and switch was run as like as real devices that they are used heavily CPU used and RAM high duty. At this same time, the author uses the HSRP system to process the redundancy. In fact, the STP (spanning tree) problem is a crucial factor that this problem can solve STP priority method. And then Active1 and Active 2 switch are a complexity problem to call same network or different phone call system. 


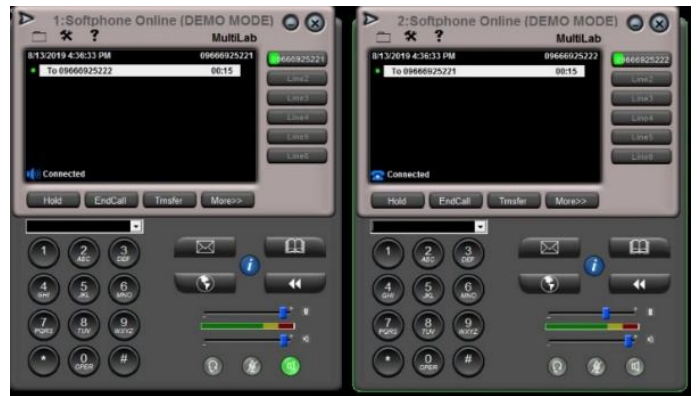

Figure 5: Phone Call in Same Network

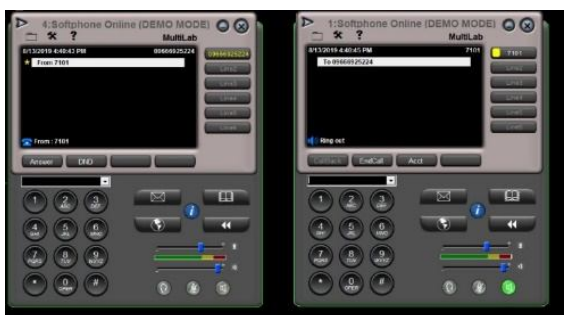

Figure 6: Phone Call in Different Network

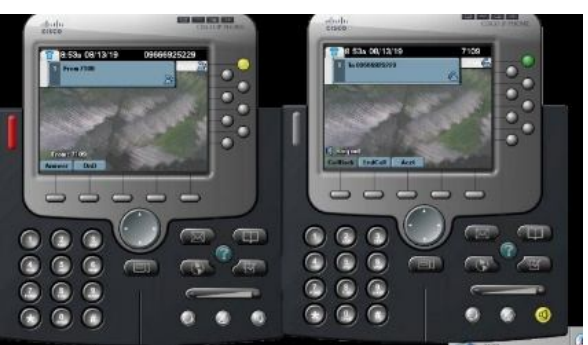

Figure 7: Phone Call in Different Network by using Cisco IP Communicator

The successfully Cacti monitoring system is shown in figure 8.

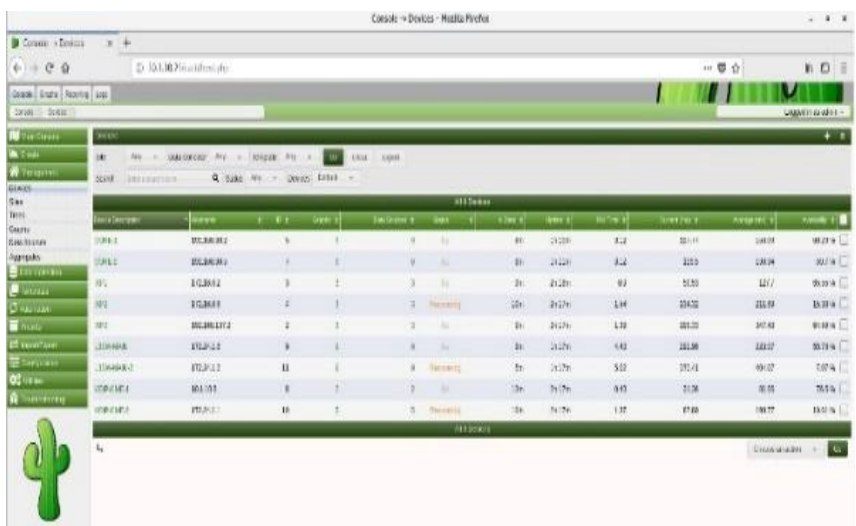

Figure 8: Cisco Device up a situation in Cacti Monitor

If the Cisco router and switch devices want to be up situation, the author should operate the time.zone synchronous and snmpwalk that it is used for to know each other such as ports and CPU usage. The three routers are used to monitor as name OSPF in figure 9.

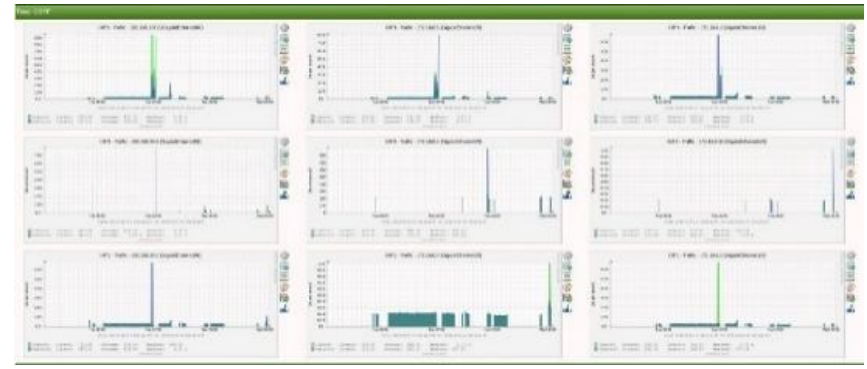

Figure 9: OSPF Network Ports monitoring

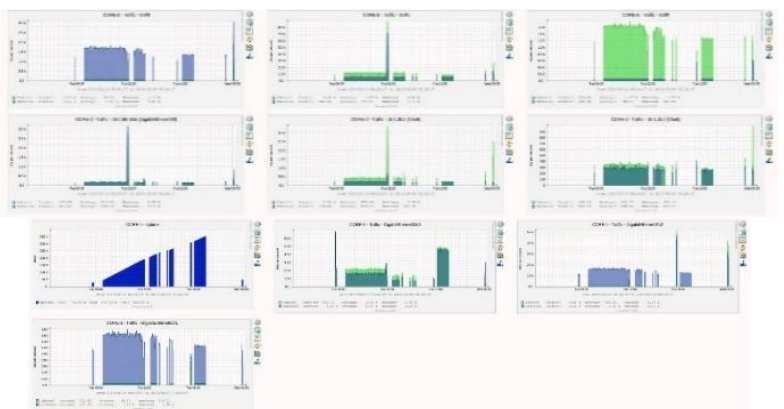

Figure 10: HSRP Network Ports monitoring

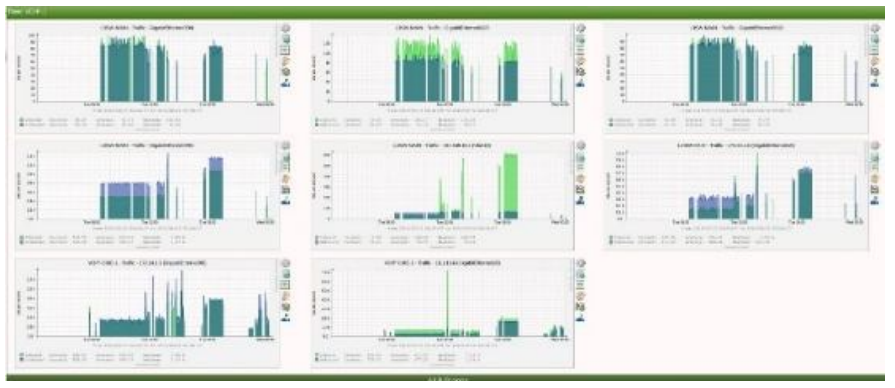

Figure 11: VoIP-1 Router Network Ports monitoring

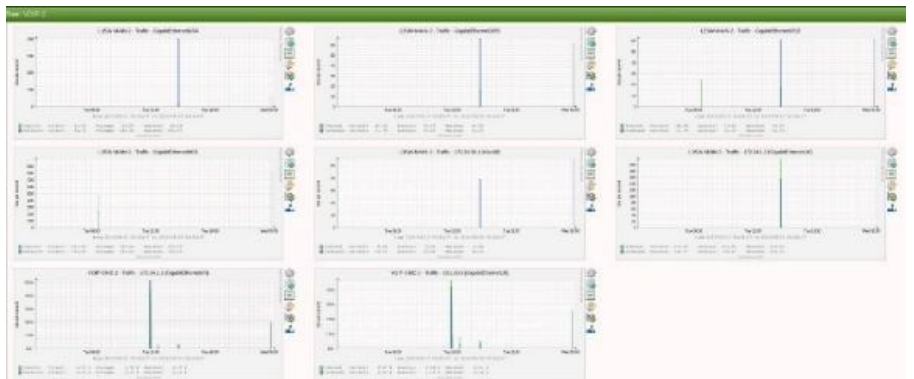

Figure 12: VoIP-2 Router Network Ports monitoring

In Figure 10, there are four L3 switches that they are operated Active and standby system. In figure 11 of VoIP- Router Network monitoring system, One CME router and two L3 switch which is used for Ether-Channel system. As the same ways, VoIP-2 router network ports monitoring system in figure 12. VoIP-1 is more used bandwidth than VoIP-2 because the VoIP-1 is used for more calling operation. VoIP-2 is less. 


\section{CONCLUSION}

The paper will reduce the cost of phone toll and mobile call. When the Internet can get anywhere, the VoIP system could use. This paper can manage and monitoring the Cisco devices in the VoIP system. In fact, the devices is heavy load or light load in network infrastructure. Moreover, this paper can also determine and know the user is crowded or not in the corresponding location. And then, the site which is breakdown or other site which is communicate can monitor and maintain or repair the site conditions.

The VoIP system can interoperate the Mac address assignment. And then it is matched IP address and Mac address. If the successful communicate with IP address and Mac- address, the voice router is assigned a phone number that it is look like used depend on Mac-address. So that, the next generation of Mobile communication will improve as ESIM that it is not used SIM card. ESIM used mobile network but is not SIM. So that, People who use many mobile SIM reduce cost effective. The technology of ESIM is used mobile phone mac-address. So that, when the user can go anywhere in local or other country, the user can use only one phone number. Furthermore, It can security and safely for the country. On the whole, the VoIP will substitution in other phone call system and cost effective reduce to the user.

\section{Acknowledgement}

This paper was strongly supported by Dr Aaron Don M. Africa, Associate Professor, Department of Electronic and Communication Engineering, GOKONGWEI College of Engineering.

I thank my colleagues from GOKONGWEI College of Engineering, De La Salle University who provided insight and expertise that greatly assisted the research.

\section{REFERENCES}

[1] Tun Tun Oo, Africa Aaron Don, " Design and Implementation of Data and Voice Redundancy and Line Aggregation for VOIP with multiple links," International Journal of Engineering \& Technology, 8 (1.6) (2019) 23-29

[2] Aaron Don M. Africa, Charleston Franklin C. Uy, " Development of a Cost-Efficient Waste bin Management System with Mobile Monitoring and Tracking ,"International Journal of Advanced Trends in Computer Science and Engineering, Volume 8, No.2, March - April 2019 https://doi.org/10.30534/ijatcse/2019/35822019

[3] Gabriel V.Iana and Valeriu Manuel Ionescu "Virtualization of VOIP laboratories" 978-1-4673-8180-2/15/\$31.00/2015 IEEE

[4] Mohamed I. Abouseda, Kenz A Bozed, Amer R. Zerek,"Comparative Study of QoS and Performance of VoIP usingMPLS-TE Network", 978-1-4673-9234-1/ 15/\$31.00 @2015 IEEE

[5] AG Jayasundara, LDSB Weerasinghe,DK Wickramanayeke, RMJCK Rajaguru ,"Design and
Implementing VoIP Network in the National Defence University of Sri Lanka",978-1-4673-7300-5/15/\$31.00 (C)2015 IE

[6] Oscar Fernando Pico Ortiz," DESIGN, IMPLEMENTATION AND PERFORMANCE VALIDATION OF AN IP BASED AERONAUTICAL TELECOMMUNICATIONS NETWORK USING SATELLITE LINKS ,"978-1-5090-2149-9/16/\$31.00 C2016 IEEE

[7] Chunhui Yuan Hongli Zhao "Implementing VoIP Voice Communication System based on Soft-switch Technology" 978-1-5090-5154-0/16 \$31.00 (C) 2016 IEEE, DOI 10.1109/CyberC.2016.70

[8] A. Africa, "A Rough Set-Based Expert System for Diagnosing information system communication networks." International Journal of Information and Communication Technology". Vol. 11, No. 4, pp. 496-512, 2017. https://doi.org/10.1504/IJICT.2017.10008315

[9] A. Africa and L. Torrizo, "Comparative Study ofIntrusion Detection Systems against Mainstream Network Sniffing Tools." International Journal of Engineering \& Technology, Vol. 7, Nos. 4.16, pp. 188-191, 2018.

[10] A. Africa, "A Rough Set Based Solar Powered Flood Water Purification System with a Fuzzy Logic Model." ARPN Journal of Engineering and Applied Sciences. Vol. 12, No. 3, pp.638-647, 2017.

[11] A. Africa, S. Bautista, F. Lardizabal, J. Patron, and A. Santos, "Minimizing Passenger Congestion in Train Stations through Radio Frequency Identification (RFID) coupled with Database Monitoring System." ARPN Journal of Engineering and Applied Sciences. Vol. 12, No. 9, pp. 2863-2869, 2017.

[12] A. Africa and J. Velasco, "Development of a Urine Strip Analyzer using Artificial Neural Network using an Android Phone." ARPN Journal of Engineering and Applied Sciences. Vol. 12, No. 6, pp. 1706-1712, 2017. 